\title{
STABILITY OF FORCED OSCILLATIONS OF A SPHERICAL PENDULUM*
}

\author{
BY \\ JOHN W. MILES** \\ University of California, Los Angeles
}

Summary. The equations of motion for a lightly damped spherical pendulum that is subjected to harmonic excitation in a plane are approximated in the neighborhood of resonance by discarding terms of higher than the third order in the amplitude of motion. Steady-state solutions are sought in a four-dimensional phase space. It is found that: (a) planar harmonic motion is unstable over a major portion of the resonant peak, (b) non-planar harmonic motion is stable in a spectral neighborhood above resonance that overlaps neighborhoods of both stable and unstable planar motions, and (c) no stable, harmonic motions are possible in a finite neighborhood of the natural frequency. The spectral width of these neighborhoods is proportional to the two-thirds power of the amplitude of excitation. The steady-state motion in the last neighborhood is quasisinusoidal (at the forcing frequency) with slowly varying amplitude and phase. The waveform, as determined by an analog computer, is periodic but quite complex.

1. Introduction. If the point of suspension of a lightly damped spherical pendulum is made to oscillate in a plane, it is observed that the steady-state response may depart from that plane in some neighborhood of resonance. The projection of the bob on a horizontal plane then may trace either a fixed ellipse, corresponding to a non-planar harmonic motion, or a quasi-ellipse of slowly, but non-uniformly, rotating axes and slowly changing shape, corresponding to a non-planar oscillation with slowly varying amplitude and phase. This last motion is reminiscent of the free oscillations of an undamped spherical pendulum [1], but in that case angular momentum is conserved, and the rotation of the quasi-ellipse is uniform.

The linearized differential equations for the transverse motions (say the Cartesian coordinates of the projection on a horizontal plane) of a spherical pendulum are uncoupled. The non-linear equations are, however, coupled, in consequence of which energy may be transferred between the two degrees of freedom. Planar motion is still a solution of those equations, to be sure, but it need not be stable with respect to transverse perturbations.

We present here an analysis based on the equations of motion obtained by including terms of first and third order in the amplitude of motion, but discarding all non-linear terms of higher order. We shall seek quasi-sinusoidal solutions (at the forcing frequency) with slowly varying amplitude and phase. This leads to the description of the motion in a four-dimensional phase space, in which simple harmonic motions-i.e., those with constant amplitude and phase - appear as singular points. We shall determine these singular points for both planar and non-planar motions and shall examine the stability of motions in their neighborhoods. Explicit solutions for phase-space trajectories that

\footnotetext{
${ }^{*}$ Received May 12, 1961. This work was carried out by the writer as Consultant, Space Technology Laboratories, Inc., and appeared originally as STL Report EM 11-2 (March, 1960).

${ }^{* *}$ Now at the Institute of Advanced Studies, Australian National University, Canberra, Australia.
} 
do not remain in the neighborhoods of the singular points appear to be beyond presently available powers of analysis, but such trajectories have been studied on an analog computer.

The essential results of the present analysis are:

a) Planar harmonic motion is unstable over a major portion of the resonant peak in an amplitude-frequency plane.

b) Non-planar harmonic motion is stable in a spectral neighborhood above resonance that overlaps neighborhoods of both stable and unstable planar motions.

c) No stable, harmonic motions are possible in a finite neighborhood of the natural frequency $(g / l)^{1 / 2}$.

The spectral width of each of these neighborhoods is proportional to the two-thirds power of the amplitude of excitation.

Acknowledgements. I am indebted to L. E. Freed for bringing this problem to my attention $\dagger$ and to W. L. Graves, R. E. Hutton and F. C. Rieman for aid with the analog computer studies.

2. Equations of motion. We consider a spherical pendulum of length $l$ and point mass $m$ suspended from the origin of the Cartesian coordinate system $(l x, l y, l z)$ in a gravitational field $(g)$ directed along the positive $z$-axis. The position of static equilibrium then is $(0,0,1)$, while the geometric constraint is

$$
x^{2}+y^{2}+z^{2}=1 .
$$

Let $\left(\frac{1}{2} \pi-\alpha, \frac{1}{2} \pi-\beta, \gamma\right)$ be the direction cosines of the pendulum, so that

$$
x=\sin \alpha, \quad y=\sin \beta, \quad z=\cos \gamma,
$$

and

$$
\sin ^{2} \gamma=\sin ^{2} \alpha+\sin ^{2} \beta .
$$

We shall use $\alpha$ and $\beta$ as the generalized coordinates of the pendulum.

Now let us suppose that this Cartesian coordinate system is subjected to the harmonic displacement $(\epsilon \cos \omega t, 0,0)$. The kinetic and potential energies and their secondorder approximations (retaining terms through fourth order in $\epsilon, \alpha$ and $\beta$ ) then are

$$
\begin{aligned}
T & =\frac{1}{2} m l^{2}\left[\left(-\epsilon \omega \sin \omega t+\alpha^{\cdot} \cos \alpha\right)^{2}+\left(\beta^{\cdot} \cos \beta\right)^{2}+\left(\gamma^{\cdot} \sin \gamma\right)^{2}\right] \\
& \doteqdot \frac{1}{2} m l^{2}\left[(\epsilon \omega \sin \omega t)^{2}-2 \epsilon \omega\left(1-\frac{1}{2} \alpha^{2}\right) \alpha^{\cdot} \sin \omega t+\alpha^{\cdot 2}+\beta^{\cdot 2}+2 \alpha \beta \alpha^{\cdot} \beta^{\cdot}\right]
\end{aligned}
$$

and

$$
\begin{gathered}
V=m g l(1-\cos \gamma) \\
V=m g l(1-\cos \gamma) \\
\doteqdot \frac{1}{2} m g l\left[\alpha^{2}+\beta^{2}+\frac{1}{2} \alpha^{2} \beta^{2}-\frac{1}{12}\left(\alpha^{4}+\beta^{4}\right)\right] .
\end{gathered}
$$

$\dagger$ Freed predicted the instability of forced, planar oscillations of an undamped spherical pendulum in an unpublished memorandum (Space Technology Laboratories, 18 October, 1957). His analysis contained certain inconsistencies, which led the writer to undertake the present analysis. 
Forming the Lagrangian $T-V$ from (2.4b) and (2.5b), we may proceed to the secondorder approximations to the equations of motion in the form

$$
L \alpha-\frac{1}{6} p^{2} \alpha^{3}+\frac{1}{2} \alpha L \beta^{2}=\epsilon \omega^{2} \cos \omega t
$$

and

$$
L \beta-\frac{1}{6} p^{2} \beta^{3}+\frac{1}{2} \beta L \alpha^{2}=0,
$$

where

$$
L=\frac{d^{2}}{d t^{2}}+p^{2}
$$

and

$$
p=(g / l)^{1 / 2} .
$$

We have neglected a factor of $\cos \alpha$ on the right-hand side of (2.6a) in anticipation of the fact that $\alpha=O\left(\epsilon^{1 / 3}\right)$ in the subsequent analysis.

We may extend the preceding formulation to include damping by introducing the appropriate dissipation function. Taking the damping force to be equal (in magnitude) to the relative velocity multiplied by the damping coefficient $2 \delta m p$, where $\delta$ is the ratio of actual damping to critical damping in first approximation, we find that we may generalize $(2.6 \mathrm{a}, \mathrm{b})$ simply by letting

$$
L=\frac{d^{2}}{d t^{2}}+2 \delta p \frac{d}{d t}+p^{2} .
$$

If the damping were proportional to the absolute velocity of the mass we also would have to add the term $2 \delta \epsilon p \omega \sin \omega t$ to the right-hand side of (2.6a), but this term would be negligible in the subsequent calculations, which assume $\delta=O\left(\epsilon^{2 / 3}\right)$.

3. Harmonic motion in plane of excitation. Setting $\beta \equiv 0$ in $(2.6 \mathrm{a}, \mathrm{b})$, we obtain

$$
L \alpha-\frac{1}{6} p^{2} \alpha^{3}=\epsilon \omega^{2} \cos \omega t \text {. }
$$

This is Duffing's equation, and its solution for $|\epsilon| \ll 1$ has been discussed extensively $[2,3]$. We shall be concerned only with that neighborhood of resonance in which $L \alpha$, $p^{2} \alpha^{3}$ and $\epsilon \omega^{2}$ are of the same order of magnitude as $\epsilon \rightarrow 0$, i.e. $\alpha=O\left(\epsilon^{1 / 3}\right)$ and $(\omega / p)^{2}=$ $1+O\left(\epsilon^{2 / 3}\right)$. We then may pose the solution to $(3.1)$ as a Fourier series in $(2 n+1) \omega t$ with amplitudes of $O\left[\epsilon^{(2 n+1) / 3}\right]$. Only the first two terms of this series need be retained within the approximations already implicit in $(2.6 \mathrm{a}, \mathrm{b})$, however, and we therefore write

$$
\alpha(t)=\epsilon^{1 / 3} a \cos \theta+\epsilon a_{3} \cos 3 \theta+O\left(\epsilon^{5 / 3}\right)
$$

and

where

$$
\omega^{2}=p^{2}\left(1+\epsilon^{2 / 3} \nu\right),
$$

$$
\theta=\omega t,
$$

$a$ and $a_{3}$ are dimensionless amplitudes, and $\nu$ is a dimensionless measure of frequency. Substituting (3.2)-(3.4) into (3.1), neglecting terms of $O\left(\epsilon^{5 / 3}\right)$, and equating the coefficients of $\cos \theta$, we obtain

$$
\frac{1}{8} a^{3}+\nu a+1=0
$$


or, equivalently,

$$
\nu=-a^{-1}-\frac{1}{8} a^{2} .
$$

The resonance curve given by (3.6) is plotted in Fig. 1. The left and right-hand

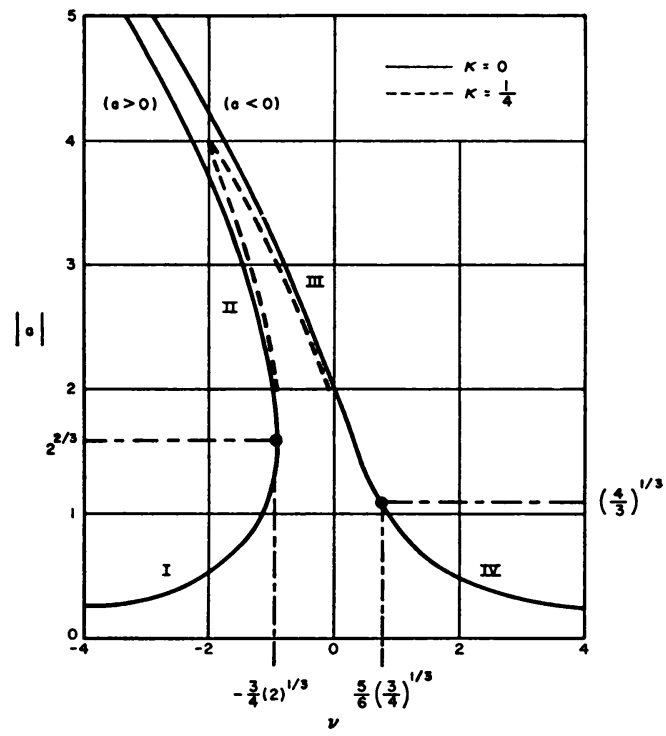

Fig. 1. The resonance curves for forced, planar oscillations of a spherical pendulum, as given by (3.6) and (3.12). Stable oscillations of the undamped pendulum are restricted to branches I and IV. The oscillations of branches II and III are unstable, although those of branch III would be stable if the motion were constrained to the plane of excitation.

branches comprise those points for which $a>0$ and $a<0$, respectively; portions II and III of these branches represent unstable motions, as we shall show in Sec. 5 below. We emphasize that (3.5) and (3.6) are not valid for large $\nu$ (since $\epsilon^{2 / 3} \nu$ has been assumed to be small) or, equivalently, for either very small $|a|$ or very large $|a|$.

The appropriate solution to (3.1) for small $|a|$ is given by the linear approximation

$$
\alpha=\epsilon\left[(p / \omega)^{2}-1\right]^{-1} \cos \theta .
$$

We remark that if $\epsilon^{2 / 3} \nu$ is added to the left-hand side of (3.5) the result (3.2) tends uniformly to (3.7) as $|a| \rightarrow 0$, while (3.6) goes over to

$$
\nu=-\left(a+\epsilon^{2 / 3}\right)^{-1}\left(1+\frac{1}{8} a^{2}\right) .
$$

The appropriate solution to (3.1) for large values of $|a|$ requires the inclusion of damping. We shall assume that $\delta=O\left(\epsilon^{2 / 3}\right)$ in (2.9) and write

We then replace (3.2) by

$$
\delta=\frac{1}{2} \kappa \epsilon^{2 / 3} \text {. }
$$

$$
\alpha=\epsilon^{1 / 3}|a| \cos (\theta-\phi)+\epsilon\left|a_{3}\right| \cos \left(3 \theta-\phi_{3}\right)+O\left(\epsilon^{5 / 3}\right),
$$

substitute into (3.1) with $L$ given by (2.9), and equate coefficients of $\cos (\theta-\phi)$ and $\sin (\theta-\phi)$ to obtain

$$
\frac{1}{8}|a|^{3}+\nu|a|^{2}+\cos \phi=0
$$


and

$$
\kappa|a|=\sin \phi .
$$

Eliminating $\phi$ between (3.11a) and (3.11b), we obtain

$$
\nu= \pm\left(a^{-2}-\kappa^{2}\right)^{1 / 2}-\frac{1}{8} a^{2}, \quad|a| \leq \kappa^{-1} .
$$

This last result, which evidently tends to (3.6) as $\kappa|a|$ tends to zero, is plotted in Fig. 1 for $\kappa=\frac{1}{4}$. The resonant peak occurs at $\nu=-1 /\left(8 \kappa^{2}\right)$ and $|a|=1 / \kappa$, where $\phi=\pi / 2$, while $\phi$ tends to 0 and $\pi$ as $\nu$ tends to $-\infty$ and $+\infty$ respectively. The peak value of $\alpha$ is $\epsilon^{1 / 3} / \kappa=\epsilon / 2 \delta$, exactly as in the linear approximation; in either approximation, this peak represents the maximum response at the forcing frequency and is characterized by a balance between applied force and damping force at a frequency determined by a balance between inertial and restoring forces.

We shall consider the stability of the foregoing solution in Sec. 5 below.

4. Differential equations in phase space. Guided by the considerations of the preceding section, we seek a general solution to $(2.6 \mathrm{a}, \mathrm{b})$ in the neighborhood of resonance-i.e., for $\left|\omega^{2}-p^{2}\right|=O\left(\epsilon^{2 / 3} p^{2}\right)$ as $\epsilon \rightarrow 0$-in the form*

$$
\alpha=\epsilon^{1 / 3}\left[f_{1}(\tau) \cos \theta+f_{2}(\tau) \sin \theta\right]
$$

and

$$
\beta=\epsilon^{1 / 3}\left[f_{3}(\tau) \cos \theta+f_{4}(\tau) \sin \theta\right],
$$

where

$$
\tau=\frac{1}{2} \epsilon^{2 / 3} \theta,
$$

and regard the $f_{i}(i=1,2,3,4)$ as Cartesian coordinates in a four-dimensional phase space. Substituting (4.1), (3.3) and (3.9) into $(2.6 \mathrm{a}, \mathrm{b})$ and (2.9) and equating coefficients of $\cos \theta$ and $\sin \theta$, we may place the results in the form

$$
\frac{d f_{i}}{d \tau}=F_{i}\left(f_{1}, f_{2}, f_{3}, f_{4}\right)-\kappa f_{i}
$$

where

$$
F_{1}=-H_{, 2}, F_{2}=H_{, 1}, F_{3}=-H_{, 4}, F_{4}=H_{, 3},
$$

and

$$
H=f_{1}+\frac{1}{2} \nu f_{i} f_{i}+\frac{1}{32}\left(f_{i} f_{j}\right)^{2}-\frac{3}{8}\left(f_{2} f_{3}-f_{4} f_{1}\right)^{2} .
$$

Repeated subscripts imply summation and subscripts following commas imply differentiation with respect to the corresponding $f_{i}$, in accord with the usual conventions for Cartesian tensors.

It is well known that, by virtue of the fact that $F_{i}-\kappa f_{i}$ is an integral function of the $f_{i}$, the set of differential equations (4.2) has a unique solution for any prescribed set of initial values, $f_{i}(0)$. The available tools of analysis appear to be inadequate for the explicit calculation of this solution for arbitrary $f_{i}(0)$, however, and we shall find it necessary to restrict the subsequent analysis primarily to a discussion of the solutions

*This is an obvious generalization of Van der Pol's method for a single degree-of-freedom oscillator. 
of (4.2) in the neighborhoods of its singular points. Some global properties of (4.2) are discussed in the Appendix, but they do not go very far toward the main goal of explicit solutions.

5. Solutions near the singular points. The singular points of the set of differential equations (4.2) are given by the zeros of the algebraic equations

$$
F_{i}-\kappa f_{i}=0 .
$$

The coordinates of such a point obviously constitute a solution to (4.2), and the substitution of these coordinates in $(4.1 \mathrm{a}, \mathrm{b})$ yields a simple harmonic motion. Conversely, the solution $(4.1 \mathrm{a}, \mathrm{b})$ can be simple harmonic only at a singular point in the phase space. [The description simple harmonic is, of course, appropriate only insofar as terms of $O(\epsilon)$ are neglected in $\alpha$ and $\beta$.]

To determine the stability of the harmonic motion corresponding to a given singular point, say $f_{i}^{(0)}$, we may consider the perturbation solution

$$
f_{i}(\tau)=f_{i}^{(0)}+c_{i} \exp [(\lambda-\kappa) \tau], \quad\left|c_{i}\right| \ll 1 .
$$

Such a solution tends to the singular point if $\lambda<\kappa$, remains in the neighborhood of the singular point if $\lambda=\kappa$, or departs from this neighborhood if $\lambda>\kappa$. We designate both the singular points and the corresponding harmonic motions as stable if $\lambda \leq \kappa$ or unstable if $\lambda>\kappa$, but we emphasize that these terms are mathematically significant only for trajectories originating in the neighborhood of the singular point under consideration. In particular, more than one stable singular point may exist for given values of $\nu$ and $\kappa$ (see below), and the asymptotic behavior (as $\tau \rightarrow \infty$ ) of a solution for these values of $\nu$ and $\kappa$ then must depend on the initial conditions $f_{i}^{(0)}$.

Substituting (5.2) into (4.2) and linearizing in the $c_{i}$, we obtain

$$
\left[\lambda \delta_{i j}-F_{i, j}^{(0)}\right] c_{i}=0,
$$

where $\delta_{i}$, is the Kronecker delta. It follows that the $\lambda$ 's must be equal to the latent roots of the matrix $\left[F_{i, i}^{(0)}\right]$. Evaluating $F_{i, j}$ from (4.3), we may place the characteristic determinant in the form

$$
\left|\lambda \delta_{i j}-F_{i, i}^{(0)}\right|=\lambda^{4}+\left(M_{1212}+2 M_{1234}+M_{3434}\right) \lambda^{2}+\left|H_{, i i}^{(0)}\right|=0,
$$

where $M_{p a r s}$ is that minor of $\left|H_{i, i}^{(0)}\right|$ containing those elements common to rows $p, q$ and columns $r, s$ (note that $M_{1234} \equiv M_{3412}$ ).

Let us consider first the stability of the harmonic solutions for planar motion. The solution to (5.1), as obtained from (3.11) and (3.12), then is given by

$$
f_{1}=a r, \quad f_{2}=\kappa a^{2}, \quad f_{3}=0, \quad f_{4}=0,
$$

and

$$
\nu=-a^{-1} r-\frac{1}{8} a^{2},
$$

where

$$
r=+\left(1-\kappa^{2} a^{2}\right)^{1 / 2}
$$

and

$$
a \gtrless 0 \text { as } \quad \begin{aligned}
& 0 \\
& \frac{1}{2} \pi
\end{aligned} \leq \phi \leq \frac{1}{2} \pi .
$$


Substituting (5.5) into (5.3), we find that either

$$
\lambda^{2}=\lambda_{\alpha}^{2}=\frac{1}{4} a^{-2} r\left(a^{3}-4 r\right), \quad c_{3}=c_{4}=0,
$$

or

$$
\lambda^{2}=\lambda_{\beta}^{2}=-\frac{3}{4} a^{-2} r\left(a^{3}+\frac{4}{3} r\right), \quad c_{1}=c_{2}=0 .
$$

The critical condition $\lambda_{\alpha}^{2}=\kappa^{2}$ implies $\dot{r} a^{3}=4$, while $\lambda_{\beta}^{2}=\kappa^{2}$ implies $r a^{3}=-4 / 3$; each of these conditions yields two real roots for sufficiently small values of $\kappa$. The roots of $\lambda_{\alpha}^{2}=\kappa^{2}$ imply $d \nu / d|a|=0$, corresponding to the points of vertical tangency on the resonance curve of Fig. 1, and that portion of the resonance curve between these two points represents unstable oscillations of the pendulum independently of whether or not it is constrained to the plane of excitation. The roots of $\lambda_{\beta}^{2}=\kappa^{2}$ occur on the righthand branch $(a<0)$ of the resonance curve and are significant only if transverse motion of the pendulum is possible.

If $\kappa=0, \lambda_{\alpha}^{2}>0$ for $a>2^{2 / 3}$ and $\lambda_{\beta}^{2}>0$ for $-a>(4 / 3)^{1 / 3}$. We designate the corressponding, unstable branches of Fig. 1 as II and III and the stable branches as I and IV. If $\kappa^{2} \ll 1$ the upper critical points are given by

and

$$
\lambda_{\alpha}^{2}=\kappa^{2} \text { at } a=\kappa^{-1}-8 \kappa^{5}+O\left(\kappa^{11}\right)
$$

and by

$$
\nu=-(1 / 8) \kappa^{-2}-2 \kappa^{4}+O\left(\kappa^{10}\right)
$$

and

$$
\lambda_{\beta}^{2}=\kappa^{2} \text { at }-a=\kappa^{-1}-(8 / 9) \kappa^{5}+O\left(\kappa^{11}\right)
$$

$$
\nu=-(1 / 8) \kappa^{-2}+(14 / 9) \kappa^{4}+O\left(\kappa^{10}\right) .
$$

We therefore arrive at the curious result that planar oscillations of a damped spherical pendulum are stable in a very small neighborhood of the resonant peak-viz., $-2 \kappa^{4}<\nu+(1 / 8) \kappa^{-2}<(14 / 9) \kappa^{4}$-but are unstable over most of the remainder of this peak-namely for $a>2^{2 / 3}+\frac{2}{3} \kappa^{2}+O\left(\kappa^{4}\right)$ below resonance and for $a>(4 / 3)^{1 / 3}+$ $(2 / 9) \kappa^{2}+O\left(\kappa^{4}\right)$ above resonance. It seems likely, however, that the initiation of a phasespace trajectory terminating at the singularity corresponding to this small neighborhood of the resonant peak would be quite difficult and that most trajectories in this frequency band would terminate on branch I (see Sec. 6).

It is relatively straightforward to show that (5.1) has at most one solution other than that of (5.5). If $\kappa=0$ this solution is given by

$$
f_{1}=a, \quad f_{2}=0, \quad f_{3}=0, \quad f_{4}^{2}=b^{2}=a^{2}+\frac{4}{3} a^{-1}
$$

and

$$
\nu=-\frac{1}{6} a^{-1}+\frac{1}{2} a^{2}
$$

provided that either $a>0$ or $a<-(4 / 3)^{1 / 3} ; b$ is imaginary if $-(4 / 3)^{1 / 3}<a<0$. Substituting (5.9) into (5.4), we obtain

$$
\lambda^{4}+\frac{9}{4} a\left(a^{3}+\frac{8}{9}\right) \lambda^{2}+\frac{9}{8} a^{-1}\left(a^{3}+\frac{1}{6}\right)\left(a^{3}+\frac{4}{3}\right)=0 .
$$


Regarding (5.10) as a quadratic in $\lambda^{2}$ and invoking Descartes' rule of signs, we see that it has one positive root if $-a^{3}>4 / 3$. This implies (since $b^{2}<0$ if $-a^{3}<4 / 3$ ) that there can be no stable harmonic motions corresponding to $a<0$ in (5.9). If $a>0$ there are no positive real roots to $(5.10)$, but the roots are complex if $a<0.841(\nu<0.154)$.

We conclude that the non-planar, undamped harmonic motion defined by (5.9) is stable only for $a>0.841(\nu>0.154)$. The locus of those stable motions is plotted as branch VI in Fig. 2, together with the stable branches I and IV for planar oscillations.

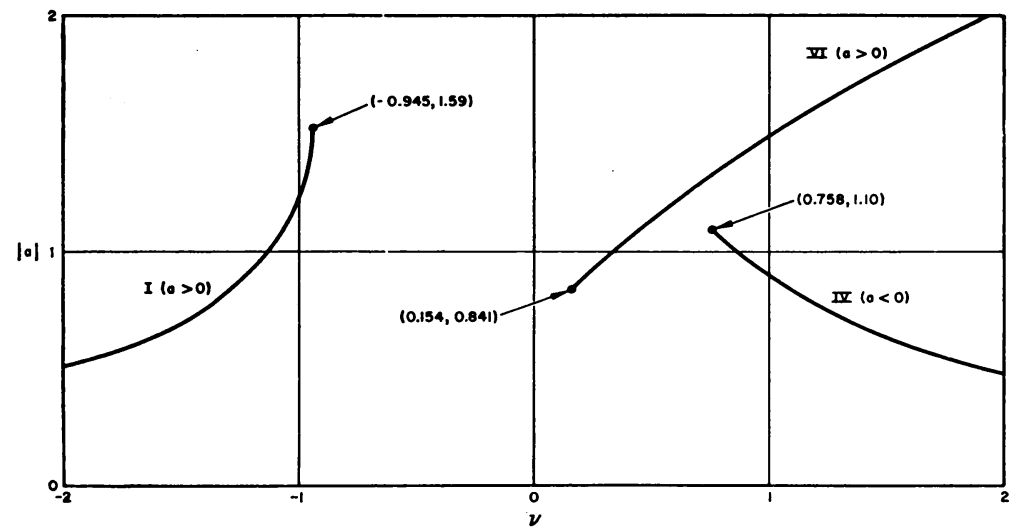

Fig. 2. Branches I and IV comprise the stable solutions for (forced) planar oscillations of a spherical pendulum and branch VI the stable solutions for non planar oscillations. See Table 1 for a summary of both stable and unstable branches.

The explicit results corresponding to (5.9) for $\kappa>0$ are quite cumbersome; accordingly, we state only the result that the maximum displacement for non-planar, harmonic motion is given by

$$
\left(\alpha^{2}+\beta^{2}\right)_{\max }^{1 / 2}=2^{-1 / 2} \epsilon^{1 / 3} \kappa^{-1}=2^{-3 / 2}(\epsilon / \delta)
$$

at

$$
f_{1}=0, \quad f_{2}=\frac{1}{2} \kappa^{-1}, \quad f_{3}=\frac{1}{2}(1+s) \kappa^{-1} \quad f_{4}=\frac{1}{2}(1-s) \kappa^{-1}
$$

and

$$
\nu=\frac{1}{32}(1+3 s) \kappa^{-2},
$$

where

$$
s=\left[1-(32 / 3)^{2} \kappa^{6}\right]^{1 / 2} .
$$

We observe that the maximum potential energy of this motion, being proportional to the maximum value of $\alpha^{2}+\beta^{2}$ in first approximation, is only one-half that of the planar oscillation (for which $\alpha_{\max }=\epsilon / 2 \delta$ and $\beta=0$ ).

The foregoing results are summarized in Table 1 on the assumption that terms of order $\kappa^{2}$ can be neglected compared with unity. The central results are:

a) Simple harmonic, planar solutions are stable if either $\nu<-0.945$ or $\nu>0.757$.

b) Simple harmonic, non-planar solutions are stable if $\nu>0.154$.

c) Simple harmonic motions are unstable if $-0.945<\nu<0.154$. 
TABLE 1

The domains of stable and unstable harmonic motions, as plotted in Figs. 1 and 2. Branches I and IV comprise stable planar motions, while branch VI comprises stable non-planar motions.

\begin{tabular}{|c|c|c|c|c|}
\hline Branch & $a$ & $\nu=\epsilon^{-2 / 3}\left(\omega^{2} / p^{2}-1\right)$ & $\alpha$ & $\beta$ \\
\hline $\begin{array}{l}\text { I } \\
\text { II } \\
\text { III } \\
\text { IV } \\
\text { V } \\
\text { VI } \\
\text { VII }\end{array}$ & $\begin{array}{c}0,1.59 \\
1.59, \kappa^{-1} \\
-\kappa^{-1},-1.10 \\
-1.10,0 \\
0,0.841 \\
0.841, \frac{1}{2} \kappa^{-1} \\
-\frac{1}{2} \kappa^{-1},-1.10\end{array}$ & $\begin{array}{c}-\infty,-0.945 \\
-\frac{1}{8} \kappa^{-2},-0.945 \\
-\frac{1}{8} \kappa^{-2}, 0.758 \\
0.758, \infty \\
-\infty, 0.154 \\
0.154, \frac{1}{8} \kappa^{-2} \\
0.758, \frac{1}{8} \kappa^{-2}\end{array}$ & $\begin{array}{c}\text { stable } \\
\text { unstable } \\
\text { stable } \\
\text { stable } \\
\text { unstable } \\
\text { stable } \\
\text { unstable }\end{array}$ & $\begin{array}{c}\text { stable } \\
\text { stable } \\
\text { unstable } \\
\text { stable } \\
\text { unstable } \\
\text { stable } \\
\text { unstable }\end{array}$ \\
\hline
\end{tabular}

This range of instability decreases with increasing damping (probably monotonically, but we have not proved this). By stable, we imply only that the motion of a lightly damped pendulum will tend to a simple harmonic motion if its trajectory through a prescribed point in the $f_{i}$-phase space (corresponding to prescribed initial conditions) passes sufficiently close to the appropriate singular point. In particular, both planar and non-planar simple harmonic motions are stable for $0.757<\nu<\frac{1}{2} \kappa^{-1}$, and which would be approached asymptotically would depend on the initial conditions (we have not proved that either would be approached, but this seems entirely plausible on physical grounds).

6. Analog computor studies. The differential equations (4.2) were programmed on an analog computer in order to gain further insight into the character of their solutions, especially in that frequency range where no stable simple-harmonic solutions exist. The damping was adjusted to give $\kappa=\frac{1}{4}$.

The solutions to (4.2) for $\nu<-0.85$ settled down quite rapidly to the solution given by branch I of Fig. 2 (the critical value of $\nu$ given by $\lambda_{\alpha}=\kappa=\frac{1}{4}$ is -0.90 , but the difference is within the precision of the computer). As anticipated, no steady state solutions were obtained in the neighborhood of the resonant peak for planar motion, even though the results of the preceding section indicate such a possibility for a $\nu$ interval of 0.01 near $\nu=-2$ (for $\kappa=\frac{1}{4}$ ).

The asymptotic solutions for $-0.85<\nu<0.10$ were independent of the initial conditions (so that the description limit cycle would be appropriate) and were periodic, but the waveforms (of $f_{1,2,3,4}$ ) were far from sinusoidal except for values of $\nu$ just below $\nu=0.10$ (see Fig. 3). This appears to be connected with the fact that the perturbation of (5.2) exhibits a pure divergence ( $\lambda$ positive real) for $\nu$ just above -0.85 and oscillatory instability $\left(\lambda^{2}\right.$ complex) for $\nu$ just below 0.10 . It suggests that a perturbation solution of (4.2) via Fourier series might be relatively efficient in the latter neighborhood, but not elsewhere. The solutions for intermediate values of $\nu$, as shown in Fig. 3, are typical of essentially non-linear phenomena- e.g., relaxation oscillations. (We note that the motion of the pendulum is sinusoidal only if the $f_{i}$ are constant.)

The planar and non-planar solutions corresponding to branches IV and VI of Fig. 2 were observed for $\nu>0.8$ and $0.1<\nu<1.9$, respectively. The motion could be transferred between these two branches throughout the range $0.8<\nu<1.9$ by introducing transient disturbances. This range naturally lies within the overlapping range of stability 


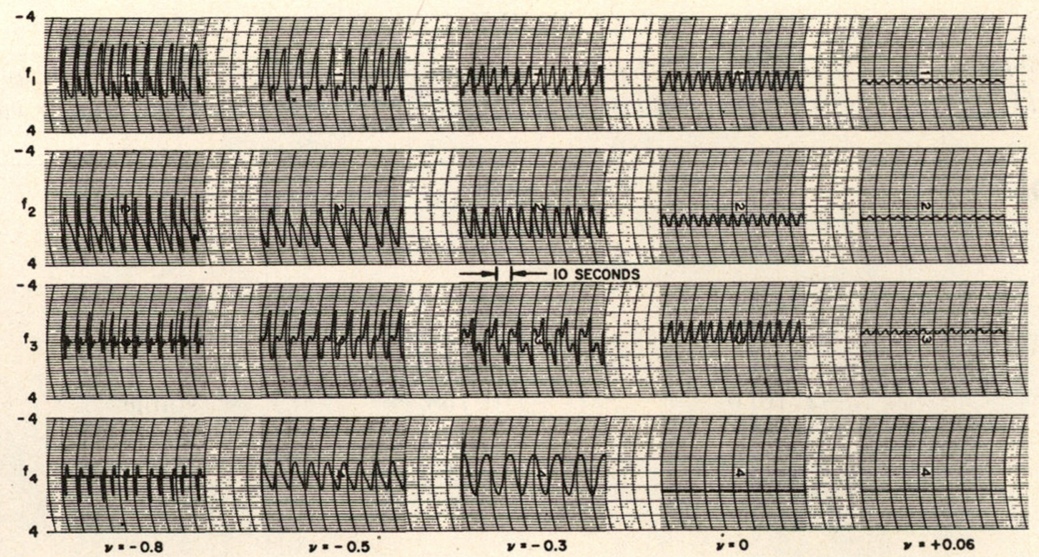

FIG. 3. The functions $f_{1,2,3,4}(\tau)$ for values of $\nu$ in the range $(-0.85$ to 0.10$)$ where no simple harmonic motions are stable.
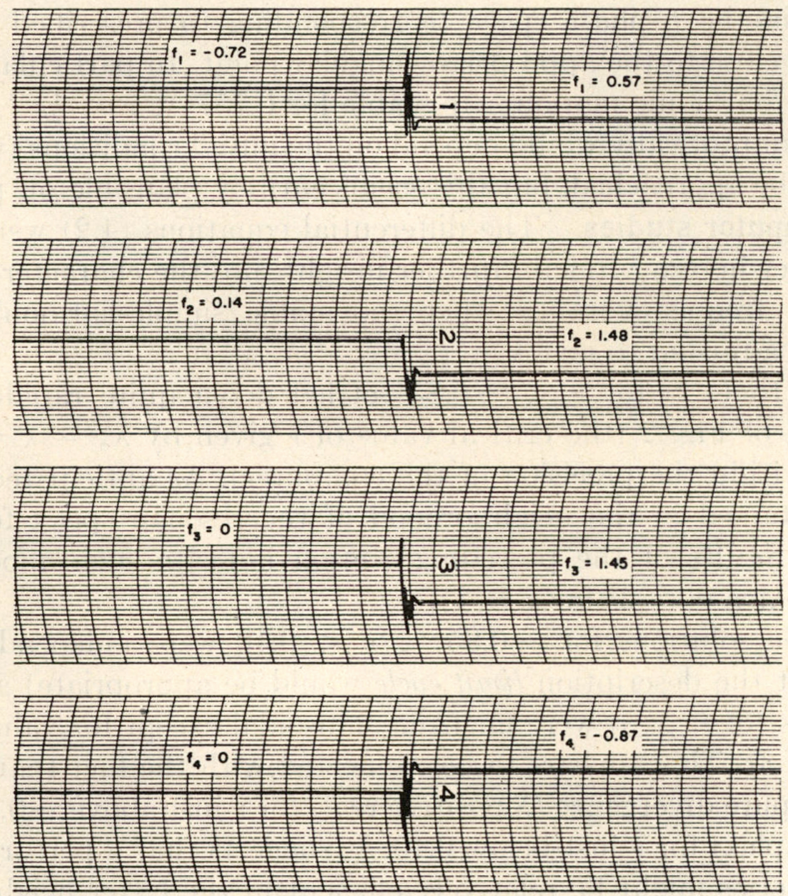

FIG. 4. The functions $f_{1,2,3,4}(\tau)$ at $\nu=1.2$, illustrating the jump (initiated by a transient disturbance) from planar to non planar, harmonic motion.

in consequence of difficulty of initiating phase-space trajectories that will terminate on branch IV as $\nu \rightarrow 0.75+$ or branch VI for $\nu \rightarrow 2-$. A typical run, illustrating the transfer from branch IV (planar motion) to branch VI (non-planar motion), is shown in Fig. 4. 
Appendix

We consider here the differential equation

$$
\frac{d f_{i}}{d \tau}=F_{i}(\mathbf{f})-\kappa f_{i},
$$

where

$$
\mathbf{f}=\left\{f_{i}\right\} \quad(i=1,2, \cdots, 2 N)
$$

is a vector in a $2 N$-dimensional phase space.

$$
F_{2 j-1}=-H_{, 2 i}, \quad F_{2 i}=H_{, 2 i-1} \quad(j=1,2, \cdots, N)
$$

and

$$
H=H(\mathbf{f}) .
$$

If $\kappa=0$ this system has the form of Hamilton's equations for a conservative system (let $f_{2 i-1}=p_{i}$ and $f_{2 j}=q_{i}$ ) with $H$ as the Hamiltonian. $\dagger$ We infer from this that

$$
H(\mathbf{f})=\text { const. } \quad(\kappa=0)
$$

is a first integral of (A1), as also may be proved directly by calculating $d H / d \tau$ with the aid of (A1) and (A3). We may extend this analogy to non-conservative systems through the transformation

$$
f_{i}=f_{i}^{*} e^{-\kappa \tau}, \quad \frac{d f_{i}^{*}}{d \tau}=F_{i}^{*}\left(f^{*}, \tau\right),
$$

where

$$
H^{*}=H^{*}(\mathbf{f}, \tau) \equiv e^{2^{\kappa \tau}} H\left(\mathbf{f} e^{-\kappa \tau}\right),
$$

but then $H^{*}=$ const. is no longer a first integral of (A6).

The result (A5) implies that a trajectory associated with the initial conditions $\mathbf{f}=\mathbf{f}_{0}$ must remain on the hypersurface $H(\mathbf{f})=H_{0}$ if $\kappa=0$, but we may prove that (A1) cannot be satisfied everywhere on a closed hypersurface if $\kappa \neq 0$. Consider the integral

$$
I=\int_{S} n_{i} \frac{d f_{i}}{d \tau} d S\left(f_{i}\right)
$$

which must vanish identically if $S$ is any closed surface in the $f_{i}$-space. If (A1) were satisfied everywhere on some $S$ we would have

$$
\begin{aligned}
I & =\int_{S} n_{i}\left(F_{i}-\kappa f_{i}\right) d S\left(f_{i}\right) \\
& =\int_{V}\left(F_{i, i}-2 N \kappa\right) d V
\end{aligned}
$$

by applying the tensor form of Gauss' divergence theorem to transform the surface integral, $V$ being the enclosed volume. But $F_{i, i} \equiv 0$ from (A3), whence (A9b) reduces to

$$
I=-2 N_{\kappa} V,
$$

†But note that $H$ of (4.4) is not the conventional Hamiltonian for the spherical pendulum. 
which can vanish only if $\kappa=0$. This result is a direct extension of the well-known criterion of Bendixson for phase planes $(N=2)$.

We may investigate the boundedness of the solutions to (A1) by forming

$$
\frac{d}{d \tau}\left(\frac{1}{2}|\mathbf{f}|^{2}\right)=f_{i} \frac{d f_{i}}{d \tau}=f_{2 j} H_{2 j-1}-f_{2 j-1} H_{2 i}-\kappa|\mathbf{f}|^{2},
$$

where $j$ is summed from 1 to $N$. In the particular case of (4.4), we obtain

$$
\begin{aligned}
\frac{d}{d \tau}\left(\frac{1}{2}|\mathbf{f}|^{2}\right)= & f_{1}-\kappa|\mathbf{f}|^{2} \\
= & \kappa\left[\left(\frac{1}{2 \kappa}\right)^{2}-\left(f_{i}-\frac{\delta_{1}^{i}}{2 \kappa}\right)\left(f_{i}-\frac{\delta_{1}^{i}}{2 \kappa}\right)\right] .
\end{aligned}
$$

It follows that the solutions to (4.2) in the phase space must tend to the interior of any hypersurface for which (A12b) is negative and hence that the asymptotic motion must be confined to the interior of a hypersphere of radius $\frac{1}{2} \kappa^{-1}$ with center at $f_{1}=\frac{1}{2} \kappa^{-1}$, $f_{2}=f_{3}=f_{4}=0$. The maximum amplitude of this asymptotic motion is given by $f_{1}=\kappa^{-1}$, $f_{2}=f_{3}=f_{4}=0$, corresponding to planar resonance.

\section{REFERENCES}

1. A. G. Webster, The dynamics of particles, Dover Publications, New York, 1959

2. J. H. Stoker, Nonlinear vibrations, Interscience Publications, New York, 1950

3. C. Hayashi, Forced oscillations in nonlinear systems, Nippon Printing and Publishing Co., Osaka, 1953

4. A. A. Andronow and C. E. Chaikin, Theory of oscillations, Princeton University Press, 1949 